\title{
Structure Characteristics of ISD Coated Conductors
}

\author{
Y. L. Tang ${ }^{1,3}$, D. J. Miller ${ }^{1}$, B. Ma ${ }^{2}$, R.E. Koritala ${ }^{2}$ and U. Balachandran ${ }^{2}$ \\ ${ }^{1}$ Electron Microscopy Center, Materials Science Division, ${ }^{2}$ Energy Technology Division, \\ ${ }^{3}$ Chemical Technology Division, Argonne National Laboratory, Argonne, IL 60439, U.S.A.
}

\begin{abstract}
The inclined-substrate deposition (ISD) method for growth of biaxially textured $\mathrm{MgO}$ is of interest due to its applications in coated conductors based on high-temperature superconductor (HTS). The ISD method is especially attractive since it offers the potential to produce a highquality biaxially textured layer in a simple and efficient process. In this work, of $\mathrm{YBa}_{2} \mathrm{Cu}_{3} \mathrm{O}_{7-\mathrm{x}}$ (YBCO) coated conductors formed by two-step deposited $\mathrm{MgO}$ buffer layers (ISD and homoepitaxial) on Hastelloy tapes were examined by high-resolution electron microscopy (HREM) carried out to study both the structure of individual layers and, especially, the interfaces between them. Special attention was paid to the $\mathrm{MgO}$ buffer layer because of its importance to biaxial YBCO formation. TEM investigation shows the $\mathrm{MgO}$ [111] direction is not parallel to the substrate normal but tilted slightly toward the deposition direction. The second layer of $\mathrm{MgO}$ has a good epitaxial relationship with the first layer, while dislocations were found near the interface area of the $\mathrm{MgO}$ layers. Twin boundaries, which are in the (111) plane, were found between the ISD MgO columns. The multi-buffer layers gave better growth of epitaxial YBCO.
\end{abstract}

\section{INTRODUCTION}

Record superconducting performance has recently been demonstrated with conductors prepared by coating thick films of YBCO onto flexible metallic substrates, and this has stimulated intense worldwide activity and expectations for realizing the full potential for operating superconducting devices at liquid nitrogen temperatures with current densities in the $\mathrm{MA} / \mathrm{cm}^{2}$ range. The key ingredient in this new development has been the ability to deposit YBCO films that are biaxially textured onto polycrystalline substrates, thus reducing the number of weak, high-angle grain boundaries that are known to limit current transport. Several techniques have been developed for preparing a biaxially textured template upon which the YBCO film can be subsequently grown epitaxially. The ion beam-assisted deposition (IBAD) process [1] achieves texture by means of a secondary ion gun that orients an oxide-film buffer layer that is being deposited onto the metallic substrate. The rolling-assisted biaxially textured substrate (RABiTS) process [2] achieves texture by mechanical rolling of a face-centered cubic metal and subsequent heat treatment. The ISD method is a simple and unique method, which was first reported by Hasegawa et al. [3,4] using excimer laser ablation. A similar biaxial alignment was observed in $\mathrm{CeO}_{2}$ and $\mathrm{MgO}$ by the ISD process [5]. At present, the critical current density $\left(\mathrm{J}_{\mathrm{c}}\right)$ values for YBCO films deposited on ISD MgO are less than those of IBAD, but ISD has very high deposition rates, which should be advantageous for scaling up toward practical applications [5-8].

The objective of this paper is to conduct detailed analytical electron microscopy on the microstructure characteristics of ISD coated conductors with different architectures. An HREM 
analysis was performed to study the micro-twin structure and the interface between the ISD $\mathrm{MgO}$ and the homo-epitaxial MgO. In addition, studies of the relationship between the buffer layer microstructure and the buffer layer diffusion barrier were conducted for various buffer layer structures.

\section{EXPERIMENTAL}

Mechanically polished Hastelloy $\mathrm{C} 276^{\circledR}$ (HC) pieces with a thickness of $\sim 0.1 \mathrm{~mm}$ were used as substrates for $\mathrm{MgO} / \mathrm{YBCO}$ deposition. The substrates were mounted above the deposition source and inclined by an angle with respect to the substrate normal. Oxygen was introduced into the deposition chamber near the substrate. The oxygen flow was adjusted so that the oxygen pressure near the e-beam evaporation source was controlled. In Samples 1 and 2, after deposition of the ISD MgO, a thin MgO layer was deposited at elevated substrate temperature and with the substrate normal to the vapor source (zero inclination angle) to improve the buffer layer surface with respect to the epitaxial YBCO growth. The homo-epitaxial growth of the MgO film led to the development of a sharp cubic texture inherited from a $\mathrm{MgO}$ film pre-deposited by e-beam evaporation, as described in detail elsewhere [8]. Sample 1 has Ag/YBCO/homo-epitaxial $\mathrm{MgO} / \mathrm{ISD}-\mathrm{MgO} / \mathrm{Hastelloy}$ architecture. Silver was deposited for protection of the film when measuring the properties and preparing the sample for transmission electron microscopy (TEM). The architecture in Sample 2 is more complicated, YSZ/ISD-MgO/Homo-epitaxial $\mathrm{MgO} / \mathrm{YSZ} / \mathrm{CeO}_{2} / \mathrm{YBCO}$ (where YSZ=yttria-stabilized zirconia). The microstructure of the thin films was investigated by analytical electron microscopy and HREM. These analyses made use of a Philips CM30-T (300KV) with an EDAX EDS system and Gatan PEELS system and JEM$4000 \mathrm{EX}$ with spherical aberration coefficient $\mathrm{Cs}=1.0 \mathrm{~mm}$. Cross-section samples for TEM examination were prepared by a method published elsewhere [9]. Cold stage was used when ion milling the sample to eliminate the temperature effect.

\section{RESULTS AND DISCUSSION}

Low-magnification TEM and HREM were conducted to reveal the overall architecture and interface structure in Samples 1 and 2. Since every interface is important to the growth quality and the transport properties, we will discuss specific interfaces separately.

\section{$\underline{\text { Overall architecture }}$}

Figure 1 shows the cross-sectional morphology of Sample 1. The grain size of $\mathrm{MgO}$ increases from $5 \mathrm{~nm}$ at the bottom to $150 \mathrm{~nm}$ near the top. The contrast in the TEM picture indicates the epitaxial and columnar growth of the ISD $\mathrm{MgO}$ film. The white contrast at the grain boundaries is revealed by HREM and selected area electron diffraction (SAED) as micro-twin structures. We

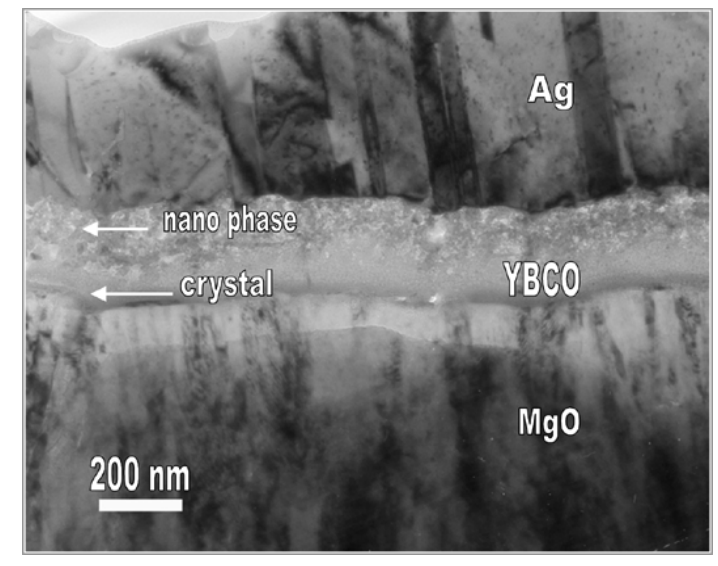

Figure 1. TEM bright-field image showing the architecture of Sample 1. The YBCO film does not form all the way to the top. There exists nano-phase structure upon the YBCO crystals. 


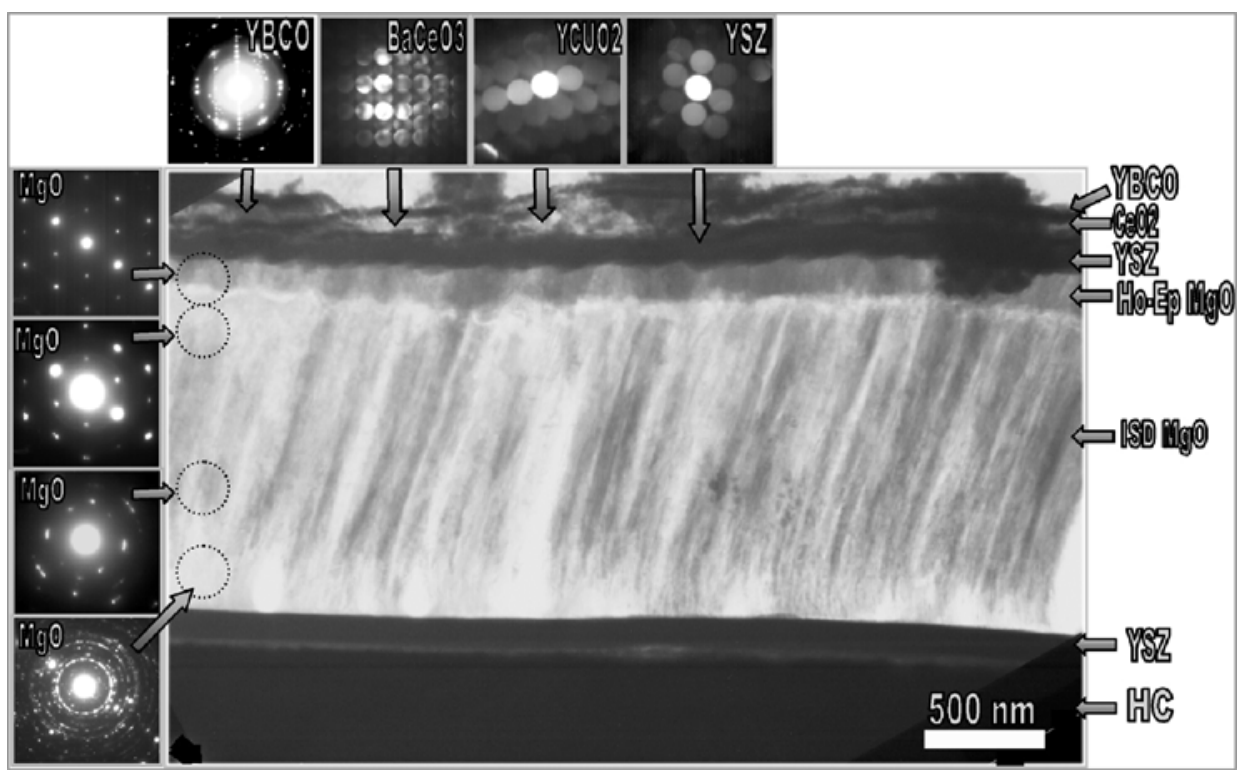

Figure 2. Cross-section TEM photographs showing the architecture of sample 2, which has a multibuffer layer structure. Diffraction patterns are from different positions or layers of the sample.

noticed that the YBCO film is not formed as a single crystal all the way to the top (near Ag). Also, the $\mathrm{MgO}$ [111] axis is not parallel to the substrate normal but tilted by an angle of $8^{\circ}$ toward the deposition direction. This phenomenon has been reported by B. Bauer et al. [10]. Figure 2 shows the architecture of Sample 2: HC/YSZ/ISD-MgO/homo-epitaxial MgO $/ \mathrm{YSZ} / \mathrm{CeO}_{2} / \mathrm{YBCO}$; the diffraction patterns are from different layers and reaction products.

\section{$\underline{\text { Hastelloy/MgO interface }}$}

The interface structure between Hastelloy and ISD MgO in Samples 1 and 2 is shown in Figure 3. This interface is clean, and no reaction phase formed. The interface is nano-scaled flat, while there is a very thin, atomically scaled amorphous layer. In Sample 2 there is a continuous reaction oxide layer formed during the process, which is similar to the phenomenon reported by Holesinger et al. [11]. A $\mathrm{Cr}_{2} \mathrm{O}_{3}$ layer is formed during PLD of the $\mathrm{CeO}_{2}$ and $\mathrm{YBCO}$ film when the substrate was heated to temperatures between $700^{\circ} \mathrm{C}$ and $800^{\circ} \mathrm{C}$, even though the first layer of YSZ was deposited at room temperature. In the ISD process, the first layer of $\mathrm{MgO}$ was formed at room temperature, while the homo-epitaxial $\mathrm{MgO}$ and $\mathrm{YBCO}$ layers were deposited at elevated temperature, which is not high enough to form an oxide layer.

\section{$\underline{\text { MgO/homo-epitaxial MgO interface }}$}

The bright contrast in the ISD MgO film in Figure 2 is from micro-twin boundaries, which is revealed by HREM and diffraction pattern [Figure 4 (a)]. The twin planes are in the (111) plane, and were found in between $\mathrm{MgO}$ columns in Sample 1 and 2. Figure 4 (a) is an example of twins in Sample 1. Figure 4 (b) shows the interface between the ISD MgO and homo-epitaxial $\mathrm{MgO}$ in sample 2. The interface is clean, without reaction phases, and the layers are in good epitaxial relationship. At the same time, high-resolution imaging revealed 

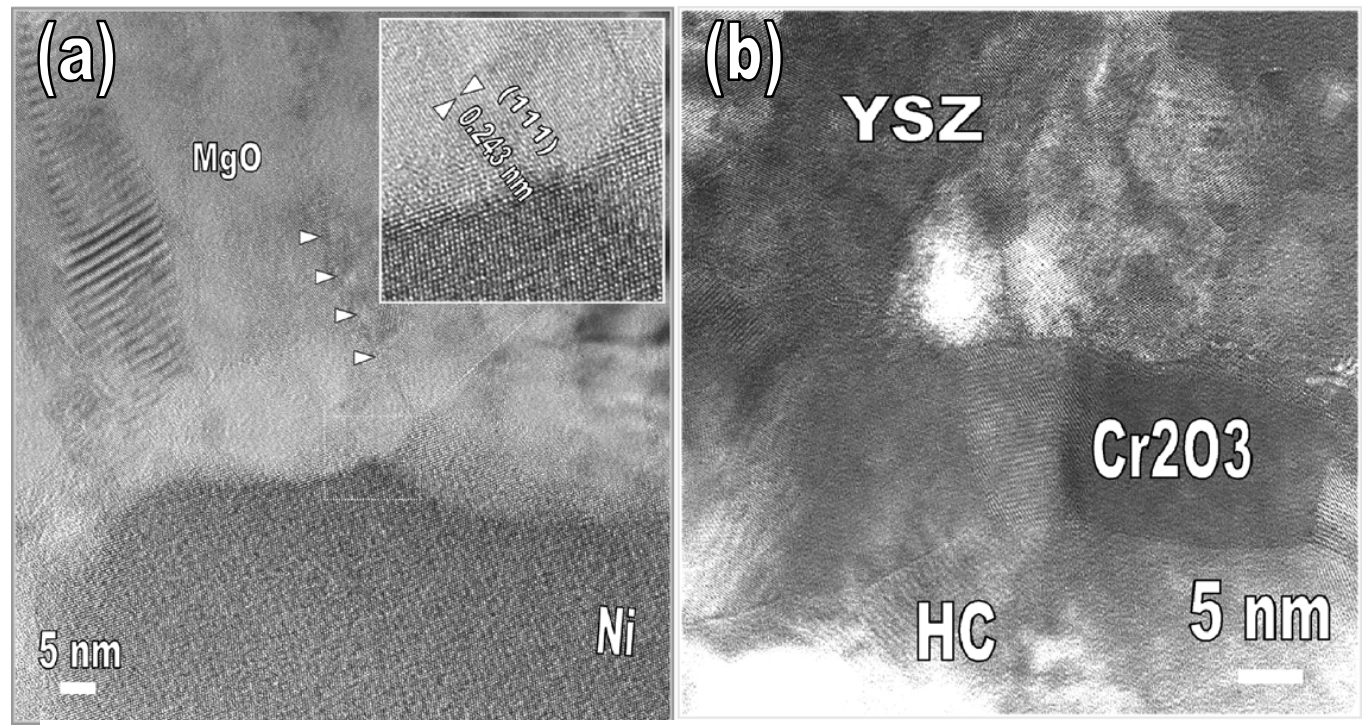

Figure 3 Hastelloy/MgO interface structure of Sample 1 (a) and Sample 2 (b) from HREM. The interface between Hastelloy and $\mathrm{MgO}$ is clean, and there is a $\mathrm{Cr}_{2} \mathrm{O}_{2}$ layer in between Hastelloy and YSZ.

the facet characteristic and dislocation structure at the interface between ISD MgO/homoepitaxial $\mathrm{MgO}$.

\section{Interface between YBCO and MgO}

Figure 5 shows the $\mathrm{YBCO} / \mathrm{MgO}$ interface structure of Sample 1 and Sample 2, respectively. In some part of Sample 1, a very thin $(10 \sim 90 \mathrm{~nm})$ YBCO layer is formed on the homo-biaxially aligned $\mathrm{MgO}$ buffer layer. The orientation relationships are $\mathrm{YBCO}[100] / \mathrm{MgO}[011]$ and $\mathrm{YBCO}[001] / / \mathrm{MgO}[111]\left(1^{\circ}\right.$ off $)$. Most of the interface is composed of reaction products, for example, $\mathrm{Ba}-\mathrm{Si}-\mathrm{Fe}-\mathrm{Ni}$ rich and $\mathrm{Si}-\mathrm{Fe}-\mathrm{Cu}$ rich phase, as detected by EDS. Severe off-c-axis YBCO is also found near $\mathrm{MgO}\left(45^{\circ}\right)$ [Figure 5 (a)]. This result indicates that an improved buffer layer should be added on top of $\mathrm{MgO}$. In Sample 2, the YBCO layer formed on a biaxially

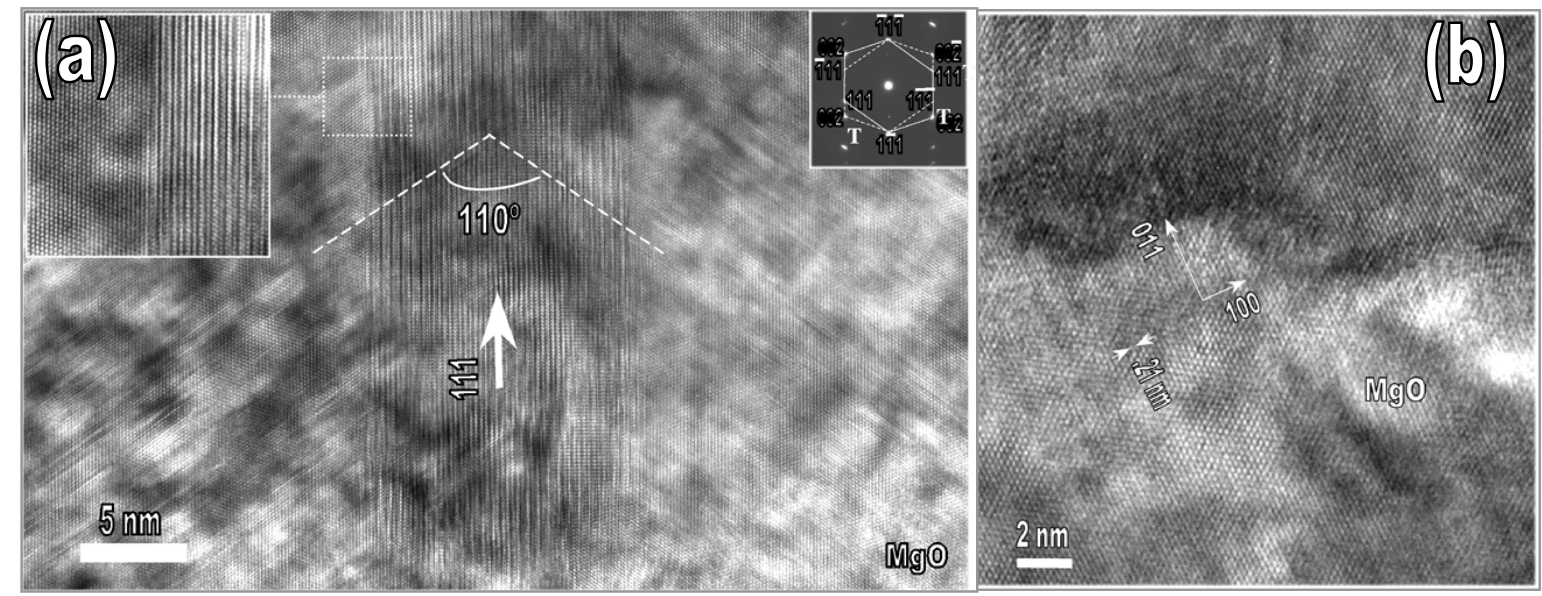

Figure 4. $\mathrm{MgO} /$ homo-epitaxial $\mathrm{MgO}$ interface structure of Sample 1(a) and Sample 2 (b) from HREM. Twins boundaries, which are in the (111) plane, were found between MgO columns in both Samples 1 and 2. 


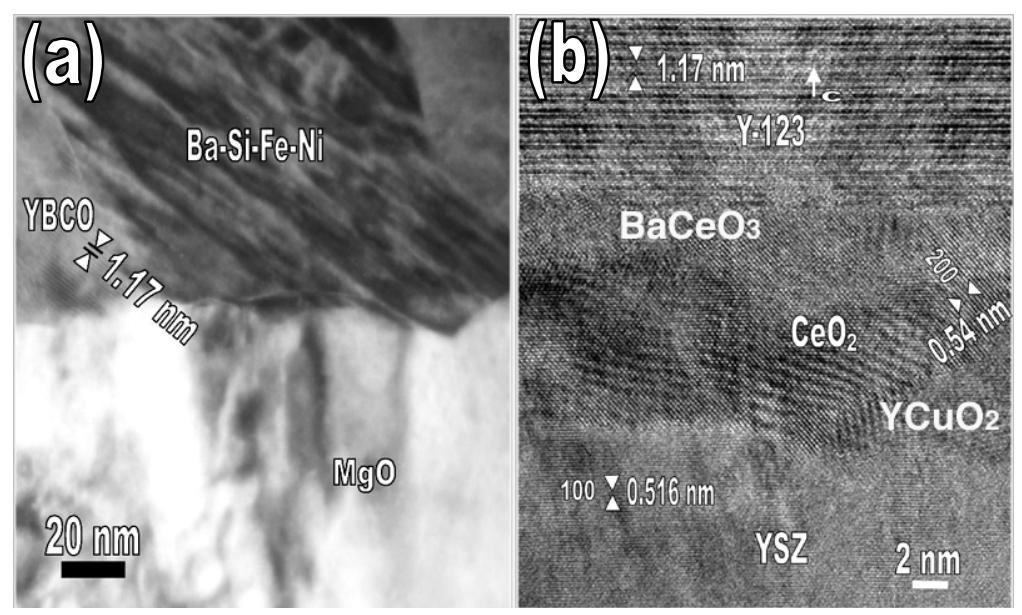

Figure 5. YBCO/MgO interface structure of Sample 1 (a) and Sample 2 (b) from HREM. In Sample 1, there is off-c-axis growth of YBCO and Ba-rich reaction phase. In sample 2, there is $\mathrm{Ba}$-rich and $\mathrm{Y}$-rich phase in between $\mathrm{CeO}_{2}$ and $\mathrm{YBCO}$.

aligned YSZ buffer layer capped with a very thin $\mathrm{CeO}_{2}$ layer. The epitaxial relationships are $\mathrm{YBCO}[001] / / \mathrm{YSZ}[001]$ and $\mathrm{YBCO}[100] / / \mathrm{YSZ}[011]$; YBCO[100]//CeO 2 [011](closely lattice matched); $\mathrm{CeO}_{2}[011] / / Y S Z[011]$ (there is misfit strain). Even though there are some reaction products in between the interface of $\mathrm{YBCO}$ and $\mathrm{CeO}_{2}$, there exists very good epitaxial relation. The reaction phases are $\mathrm{BaCeO}_{3}$ and $\mathrm{YCuO}_{2}$. Holesinger et al. [11] reported the reaction mechanism as $(4) \mathrm{CeO}_{2}+(2) \mathrm{YBCO} \Leftrightarrow(4) \mathrm{BaCeO}_{3}+(2) \mathrm{YCuO}_{2}+(4) \mathrm{CuO}+\mathrm{O}_{2}$. Since the buffer layer is almost the same as the IBAD layer of Holesinger et al. [11], we propose this as the mechanism for the ISD process in the present study. As stated above, even though the interfaces between ISD $\mathrm{MgO}$ and homo-epitaxial $\mathrm{MgO}$ are similar in both Sample 1 and Sample 2, YBCO films with better in-plane epitaxy have been demonstrated on $\mathrm{CeO}_{2} / \mathrm{YSZ}$ double-buffer layers compared to that on $\mathrm{MgO}$ buffer layers.

\section{CONCLUSIONS}

ISD is a promising method for preparing coated conductors because of the higher deposition rate and good epitaxial structure. A detailed microstructure study of ISD-coated conductors was undertaken to better understand the deposition phenomenon. The architecture of the conductor includes a YBCO thin film deposited on a biaxially textured 2- $\mu \mathrm{m}$-thick $\mathrm{MgO}$ template layer, which was fabricated by ISD on Hastelloy-C tapes. A homo-epitaxial $\mathrm{MgO}$ film was deposited on the ISD $\mathrm{MgO}$ for the purpose of increasing the epitaxial quality. The interface between the Hastelloy and $\mathrm{MgO}$ is absent of cracks and porosity and free of reaction products. On the other hand, a $\mathrm{Cr}_{2} \mathrm{O}_{3}$ phase formed between the Hastelloy and YSZ. High-resolution imaging revealed the facet and dislocation structure at the interface of ISD MgO/homo-epitaxial MgO. It also revealed the reaction products in different samples. The multibuffer layer structure could induce better epitaxial growth of YBCO, even though reaction products appear between $\mathrm{CeO}_{2}$ and YBCO. 


\section{AKNOWLEGDGMENT}

The TEM analysis was conducted in the Electron Microscopy Center at Argonne National Laboratory. The authors are grateful to Jon Hiller for assistance in TEM sample preparation. This work was supported by the U.S. Department of Energy (DOE), Energy Efficiency and Renewable Energy, as part of a DOE program to develop electric power technology, under Contract No.W-31-109-ENG-38.

\section{REFERENCES}

1. Y. Iijima, N. Tanabe, O. Kohno, and Y. Ikeno, Appl. Phys. Lett. 60, 769 (1992).

2. A. Goyal, D.P. Norton, J.D. Budai, M. Paranthaman, E.D. Specht, D.M. Kroeger, D.K. Christen, Q. He, B. Saffian, F.A. List, D.F. Lee, P.M. Martin, C.E. Klabunde, E. Hartfield, and V.K. Sikka, Appl. Phys. Lett. 69 (12), 1795 (1996).

3. K. Hasegawa, N. Yoshida, K. Fujino, H. Mukai, K. Hayashi, K. Sato, T. Ohkuma, S. Honjo, H. Ishii, and T. Hara, Proc. ICEC16/ICMC (Kitakyushu, Japan), ed., T. Haruyama, T. Mitsui, and K. Yamafuji (Tokyo: Elsevier) pp. 1413, 1997.

4. K. Hasegawa et al., Advances in Superconductivity, Vol 9, ed., S. Nakajima and M. Murakami (Tokyo: Springer), pp. 745, 1997

5. M. Bauer, J. Schwachulla, J. Egly, P. Berberich and H. Kinder, Advances in Superconductivity, Vol 10, ed., K. Osamura and I. Hirabayashi (Tokyo: Springer), pp. 979-80, 1998

6. R.E. Koritala, M.P. Chudzik, Z. Lou, D.J. Miller, C.R. Kannewurf, and U. Balachandran, IEEE Trans. Appl. Supercond. 11, 3473-3476 (2001).

7. B. Ma, M. Li, Y.A. Jee, B.L. Fisher, and U. Balachandran, Physica C, 2001 (in press).

8. H.G. Lee, Y.M. Lee, H.S.Shin, C.H.Jung, D. Youm, and G.W. Hong, Supercond. Sci. Technol. 13, 1368 (2000).

9. D.J. Miller, Midwest Society of Electron Microscopy Meeting, Argonne, March 1991.

10. M. Bauer, R. Semerad, and H. Kinder, IEEE Trans. on Applied Supercond. 9(2), 1502,(1999).

11. T. G. Holesinger, S.R. Foltyn, P. N. Arendt, H. Kung, Q.X. Jia, R. M. Dickerson, P.C. Dowden, R.F. Depaula, J. R. Groves, and J. Y. Coulter, J. Mater. Res. 15(5), 1110 (2000). 